\title{
The fermion probability amplitude as a trace over spinor indices
}

\section{MEKHFI.Mustapha ${ }^{1}$}

Département de Physique, Université Es-Senia

31100 Oran, Algérie

mekhfi@gmail.com

We re express the fermion's probability amplitude as a trace over spinor indices but using the spin formalism. Our spin formulation is appropriate to the computation of dipole moments, electric or magnetic for which we have developed such formalism. There is however a formulation of the same idea in the helicity formalism but of no direct use to us. We test the power of the trace formula by analytically computing the quark dipole magnetic moment in few lines compared to the direct computation using spinor components. The trace formulation has the advantage of making the amplitudes or even the squared amplitudes easily computable either analytically or symbolically.

European Physical Society Europhysics Conference on High Energy Physics EPS-HEP 2009, Krakow, Poland

July 16-22, 2009

\footnotetext{
$1 \quad$ Speaker
} 


\section{The amplitude as a trace}

Cross- sections and lifetimes being the squared modulus of amplitudes are traces

$$
\operatorname{Tr}\left(\bar{f} \frac{\not)^{\prime}+m}{2 m} \frac{1+\gamma_{5} \not \not^{\prime}}{2} f \frac{\not \supset+m}{2 m} \frac{1+\gamma_{5} \not \supset}{2}\right)
$$

Observables such as form factors, magnetic (electric) dipole moments etc, are probability amplitudes and are usually not expressed as traces (momenta are not paired to form projectors). In this paper we propose to rewrite the amplitude as a trace

$$
\begin{aligned}
& \bar{u}_{\alpha^{\prime}}\left(p^{\prime}, s^{\prime}\right) f_{\alpha^{\prime} \alpha}\left(s^{\prime}, s\right) u_{\alpha}(p, s)=f_{\alpha^{\prime} \alpha}\left(s^{\prime}, s\right) u_{\alpha}(p, s) \bar{u}_{\alpha^{\prime}}\left(p^{\prime}, s^{\prime}\right) \\
& =\operatorname{Tr}(f \rho), \quad \rho_{\alpha \alpha^{\prime}}=u_{\alpha}(p, s) \bar{u}_{\alpha^{\prime}}\left(p^{\prime}, s^{\prime}\right)
\end{aligned}
$$

The amplitude is then rewritten in term of the generalized spin density matrix $\rho$. To find $\rho$ we directly relate it to its form in the rest frame $\left.\rho\right|_{r f}$; calculate the latter then boost it again to the initial frame.

$$
\begin{aligned}
& u(k, s)=\exp \left(-\frac{\omega}{2} \gamma_{0} \frac{\vec{\gamma} \cdot \vec{k}}{|\vec{k}|}\right)\left(\begin{array}{c}
\chi_{s} \\
0
\end{array}\right), \quad \bar{u}(k, s)=\left(\chi_{s}^{\dagger}, \quad 0\right) \exp \left(-\frac{\omega}{2} \gamma_{0} \frac{\vec{\gamma} \cdot \vec{k}}{|\vec{k}|}\right) \gamma_{0} \\
& \left.\rho\right|_{r f}=\chi_{s}(\vec{\zeta}) \tilde{\chi}_{s^{\prime}}^{\dagger}\left(\vec{\zeta}^{\prime}\right)=\left(\frac{1+s \vec{\zeta} \cdot \vec{\sigma}}{2}\right)\left(\frac{1+s^{\prime} \vec{\zeta}^{\prime} \cdot \vec{\sigma}}{2}\right)
\end{aligned}
$$

After lengthy but straightforward calculations we get the expression for $\rho$ :

$$
\begin{aligned}
\rho\left(k, s, k^{\prime}, s^{\prime}\right) & =\left(\frac{\not k+m}{2 m}\right)\left(\frac{1+\gamma^{5} \not}{2}\right) \Re\left(\frac{k^{\prime}+m}{2 m}\right)\left(\frac{1+\gamma^{5} \not \phi^{\prime}}{2}\right) \\
\Re & =\sqrt{\frac{m m^{\prime}}{\left(k_{0}+m\right)\left(k_{0}^{\prime}+m^{\prime}\right)}}\left(1+\gamma_{0}\right)
\end{aligned}
$$

This expression can further be arranged in a covariant form which in addition does not depend on specific representations of the Dirac matrices. To this end we introduce the additional time-like vector $p=(1, \overrightarrow{0})$ and write the final form of $\Re$ as.

$$
\Re=\sqrt{\frac{m m^{\prime}}{(p k+m)\left(p k^{\prime}+m^{\prime}\right)}}(\not p+1)
$$

Our trace formulation of the amplitude in the spin framework is well adapted to the computation of the dipole magnetic or electric moments of the quarks inside baryons compared to the calculation of the same quantities using the explicit spinor components [1][2]. There is however a similar trace formulation[3] but in the helicity framework .Such a formulation although competitive to our is not adequate to dipole moment calculations as the latter ones are interpreted at the end in terms of longitudinal and 
transverse spin structure functions. We can of course retrieve all results of the helicity formalism as particular cases of the spin formalism .

\section{Application: The convection current part of the quark dipole magnetic moment}

By using the Gordon decomposition we divide the dipole magnetic moment expression into two terms, one is the convection current part and the other is the spin part. In the application of our trace formalism we only compute the convection part for illustartion of the power of the formulation. The convection part has the form:

$$
\int[\operatorname{Tr} \vec{\nabla} \rho]_{\vec{q}=0} \times \vec{k} \frac{d^{3} k}{(2 \pi)^{3}}
$$

In the above calculation we use the identities $\vec{\nabla}_{k} k_{0}=\frac{\vec{k}}{k_{0}}$ and $\vec{\nabla}_{k}|\vec{k}|=\frac{\vec{k}}{|\vec{k}|}$ to eliminate all differentiation leading to terms proportional to $\vec{k}$ as we have to take the cross product with $\vec{k} \operatorname{in}(5)$. In particular any differentiation of the spin variable is eliminated as $s$ is function of $\vec{\xi}$ and of the boost parameter $\omega=-\tanh ^{-1}\left(\frac{|\vec{k}|}{k_{0}}\right)$, idem for the primed spin. With this remark only the variable $\not k$ and $k^{\prime}$ are differentiated and we get

$$
\begin{aligned}
& -\frac{1}{2} \frac{1}{\left(k_{0}+m\right)} \int\left(\vec{k} \times \operatorname{Tr}\left[\left(\frac{k+m}{2 m}\right)\left(\frac{1+\gamma_{5} \not}{2}\right)\left(\gamma_{0} \vec{\gamma}\right)\right]\right) \frac{d^{3} k}{(2 \pi)^{3}} \\
& =\frac{1}{8 m} \frac{1}{\left(k_{0}+m\right)} \int\left(\vec{k} \times \operatorname{Tr} \gamma_{5} k \gamma_{8} \gamma_{0} \vec{\gamma}\right) \frac{d^{3} k}{(2 \pi)^{3}} \\
& =-\frac{1}{2 m} \frac{1}{\left(k_{0}+m\right)} \int|\vec{k}|^{2} \vec{s}_{\perp} \frac{d^{3} k}{(2 \pi)^{3}} \\
& =-\frac{x}{(x+1)} \int \frac{|\vec{k}|^{2}}{2 m^{2}} \vec{s}_{\perp} \frac{d^{3} k}{(2 \pi)^{3}}
\end{aligned}
$$

In the equation above, we approximate the factor $k_{0}$ by the average value of the relativistic quark energy inside the nucleon and keep the notation $k_{0}$ to designate the average value. Inserting the missing factors $\mu=\frac{Q}{2 m}$ and $\sqrt{\frac{m}{k_{0}}}$ for each spinor $\mathrm{u}$ in(5) to complete the definition of the magnetic moment and define the parameter $x=\frac{m}{k_{0}}$ we get 
the convection current part in terms of the longitudinal spin $\vec{S}$ and the transverse spin $\vec{\delta}$ :

$$
\begin{aligned}
& \frac{x \mu}{(x+1)}\left(\vec{S}-\frac{\vec{\delta}}{2 x}\right) \\
& \mu=\frac{Q}{2 m}
\end{aligned}
$$

Compare the gain of time and effort in obtaining this contribution with the lengthy method that used the components of the Dirac spinor in one of our previous work [1]

\section{Conclusion}

Dipole moments, electric or magnetic are naturally expressed in terms of the spin( longitudinal and transverse) of the quarks inside the parent baryons. Our trace formulation of the amplitude in the spin framework (as opposed to helicity formulation) is appropriate to such computations. On the other hand our formulation has the advantage of making the amplitudes or even the squared amplitudes easily computable either analytically or symbolically.

\section{References}

[1] M.Mekhfi, Correct use of the Gordon decomposition in the calculation of nucleon magnetic dipole moments, Phys.Rev.C 78 (055205) 08.

[2] Di Qing,Xiang-Song Chen,and Fan Wang, Is nucleon spin structure inconsistent with the constituent quark model Phys.Rev.D 58 (114032)1998

[3] R.Vega and J.Wudka Phys.Rev. D53 (1996) 5286-5292; Erratum-ibid. D56 (1997) 6037-6038 